\title{
Expanding Access to Medications for Opioid Use Disorder: Program and Policy Approaches from Outside the Veterans Health Administration
}

\author{
Kelsey C. Priest, PhD, MPH ${ }^{\top}$, Dennis McCarty, PhD², and Travis I. Lovejoy, PhD, MPH 2,3,4 \\ 'School of Medicine, MD/PhD Program, Oregon Health \& Science University, Portland, OR, USA; ${ }^{2}$ School of Public Health, Oregon Health \& Science \\ University-Portland State University, Portland, OR, USA; ${ }^{3}$ Department of Psychiatry, Oregon Health \& Science University, Portland, OR, USA; ${ }^{4}$ Center to \\ Improve Veteran Involvement in Care, VA Portland Health Care System, Portland, OR, USA.
}

To mitigate morbidity and mortality of the drug-related overdose crisis, the Veterans Health Administration (VHA) can increase access to treatments that save lives-medications for opioid use disorder (MOUD). Despite an increasing need, MOUD continues to be underutilized due to multifaceted barriers that exist within broader macro- and microenvironments. To promote MOUD utilization, policymakers and healthcare leaders should (1) identify and implement person-centered MOUD delivery systems (e.g., the Medication First Model, community-informed design); (2) recognize and address MOUD delivery gaps (e.g., the Best-Practice in Oral Opioid Agonist Collaborative); (3) broaden the definition of the MOUD delivery system (e.g., access to MOUD in nonclinical settings); and (4) expand MOUD options (e.g., injectable opioid agonist therapy). Increasing access to MOUD is not a singular fix to the overdose-related crisis. It is, however, a possible first step to mitigate harm, and save lives.

J Gen Intern Med 35(Suppl 3):S886-S90

DOI: $10.1007 / \mathrm{s} 11606-020-06266-3$

(c) Society of General Internal Medicine 2020

$\mathrm{D}$ espite decades of availability, access to lifesaving medications for opioid use disorder (MOUD) such as firstline opioid agonist therapy (OAT) (i.e., methadone and buprenorphine) and extended-release naltrexone is sub-optimal. Within the Veterans Health Administration (VHA), only $38 \%$ of OUD patients receive MOUD, with significant variation in facility delivery (provided in 3 to $74 \%$ of facilities). ${ }^{1}$ Barriers to MOUD outside the VHA are well described and include behaviors and attitudes of providers (e.g., stigma), treatment delivery organizations (e.g., non-patient centered systems of care), regulatory, financial, and values-related barriers. $^{2}$ Additional obstacles to MOUD utilization within the VHA include variation in facility delivery and diminished methadone capacity across the system. ${ }^{3}$ Specific sub-groups

Received August 7, 2020

Accepted September 21, 2020

Published online November 3, 2020 of patients, moreover, are less likely to receive MOUD due to institutionalized structural and interpersonal racism, ${ }^{4,5}$ classism, ${ }^{5}$ stigma, ${ }^{6}$ discrimination, ${ }^{6}$ and criminalization. For example, VHA researchers observed that patients who were older or Black had a lower odds of initiating buprenorphine. ${ }^{7}$

In this commentary, we highlight programs, models of care, and policies that may counteract institutional, regulatory, and values-related barriers to MOUD. We propose that clinical, institutional, and policy decisionmakers consider four elements to increase MOUD access and strengthen and extend the MOUD care continuum: (1) identify and implement person-centered MOUD delivery systems; (2) recognize and address MOUD delivery gaps; (3) broaden the definition of the MOUD delivery system; and (4) expand MOUD options. These elements are summarized in Table 1.

\section{ELEMENT 1: IDENTIFY AND IMPLEMENT PERSON- CENTERED MOUD DELIVERY SYSTEMS}

Systems of care that are "person-centered" (i.e., patient-centered) require the development, often co-created with patients, of organizational (e.g., culture) and provider-focused interventions (e.g., respectful and compassionate care) to enhance access to care and improve patient-reported outcomes. ${ }^{8}$ Personcentered approaches should extend beyond the walls of the healthcare setting to include policy and regulation development. A recent scoping review describes four key elements of patientcentered care defined by people with drug use disorders: (1) a therapeutic alliance (i.e., empathy and non-judgmental clinical care); (2) shared-decision making; (3) individualized care; and (4) holistic care (i.e., integration of substance use, health, and psychosocial services). ${ }^{9}$ Unfortunately, most MOUD-treatment delivery systems and policies are not grounded in personcentered approaches. We highlight two exceptions, which reflect patient centered philosophies or tactics: the Medication First (Med First) Model $^{10}$ and culturally specific MOUD delivery design for Black patients with OUD. ${ }^{11}$

The Missouri Med First program, modeled on the lowbarrier Housing First approach, ${ }^{12}$ follows four key principles: (1) patients receive MOUD as quickly as possible; (2) MOUD is available without arbitrary tapering or time limits; (3) 
Table 1 Expanding Access to Medications for Opioid Use Disorder (MOUD)

\begin{tabular}{|c|c|c|}
\hline Element & Concept & Example(s) \\
\hline $\begin{array}{l}\text { 1. Identify and implement } \\
\text { person-centered approaches } \\
\text { to MOUD }\end{array}$ & $\begin{array}{l}\text { Center and prioritize the needs of patients and } \\
\text { people who use drugs when designing MOUD } \\
\text { policies and systems of care, using a person-centered } \\
\text { philosophy. Address culturally specific needs for } \\
\text { marginalized populations with } \\
\text { intersecting risks due structural and interpersonal } \\
\text { oppression (e.g., racism). }\end{array}$ & $\begin{array}{l}\text { - Med First Model } \\
\text { - Bellevue Hospital holistic addiction clinic }\end{array}$ \\
\hline $\begin{array}{l}\text { 2. Recognize and address delivery } \\
\text { gaps in MOUD }\end{array}$ & $\begin{array}{l}\text { Identify local system gaps with the intention of } \\
\text { developing a high-touch service delivery system that } \\
\text { ensures rapid and low-barrier access to MOUD } \\
\text { within all care delivery environments. }\end{array}$ & $\begin{array}{l}\text { - The BOOST Collaborative } e^{16} \\
\text { - Addiction consult services } \\
\text { - Bridge clinics } \\
\text { - Emergency department interventions }\end{array}$ \\
\hline $\begin{array}{l}\text { 3. Broaden the definition of the } \\
\text { MOUD delivery system }\end{array}$ & $\begin{array}{l}\text { Think beyond conventional clinical settings when } \\
\text { defining and cataloging the MOUD delivery system. } \\
\text { Clinical environments may not be inviting or safe for } \\
\text { people who use drugs; thus, the availability of } \\
\text { MOUD in other spaces is critical to increase access. }\end{array}$ & $\begin{array}{l}\text { - Buprenorphine initiation through street medicine } \\
\text { programming } \\
\text { - Co-locating MOUD in harm reduction spaces, such } \\
\text { as syringe exchange programs } \text { s }^{32}\end{array}$ \\
\hline 4. Expand MOUD options & $\begin{array}{l}\text { Learn from international experiences (e.g., Canada) } \\
\text { regarding the use of other OAT options, pharmacy } \\
\text { delivery of methadone, and safe supply prescribing. }\end{array}$ & $\begin{array}{l}\text { - Broader OAT care continuum } \\
\text { - Safe supply program } \\
\text { 39 }\end{array}$ \\
\hline
\end{tabular}

individualized psychosocial services are offered but not required; and (4) MOUD is only discontinued if the patient's clinical condition deteriorates. ${ }^{10}$ The Missouri State Targeted Response (STR) implementation team conditioned STR funding on organizational capacity and willingness to deliver MOUD using Med First principles. ${ }^{10}$ Preliminary findings are promising. In the 38 sites using the Med First model, MOUD use increased from 45 to $85 \%$ of site participants, the timeliness of MOUD receipt improved (median of 0 days waiting vs. 8 days), treatment retention improved, and the median treatment cost per month declined $21 \%$ from the prior year. ${ }^{13}$

Broad sweeping low-barrier access to MOUD is not enough. Disproportionate barriers to care persist for marginalized communities, due to broader structural and oppressive forces, and the racialized two-tiered OAT system ${ }^{14}$; community-informed interventions are required. A SAMHSA report ${ }^{11}$ on the opioid-related overdose crisis for Black patients describes increasing overdose deaths in Black communities, and illustrates how community-informed strategies can enhance MOUD access. Community informants recommend that health systems and treatment programs (a) use community-led needs assessments (e.g., what matters to the community?); (b) conduct routine check-ins with the community (e.g., what is working for the community?); and, importantly, (c) explore and understand community-based assets (e.g., what are the community's strengths?). ${ }^{11}$ The report highlighted the Bellevue Hospital holistic addiction clinic, which was specifically created to meet the needs of Black patients with OUD in New York City. ${ }^{11}$ Intentional design was reflected in the physical space (e.g., "home-like"), the programming (e.g., structured cognitive behavioral therapy based on creative arts and spirituality), and organizational leadership (e.g., patient governance and intentional linkages with the community). ${ }^{11}$

The VHA system was created and designed specifically with the Veteran in mind, thus, provides a rich organizational environment for further developing and implementing personcentered MOUD systems of care. Moreover, the VHA has demonstrated interest in implementing and evaluating patientcentered care programming with the dedication of resources in the Center for Evaluating Patient-Centered Care. ${ }^{15}$ The patient-centered programing examples we provide are potential opportunities to enhance VHA MOUD-related care for sub-populations of Veterans.

\section{ELEMENT 2: RECOGNIZE AND ADDRESS MOUD DELIVERY GAPS}

Improving access to MOUD within healthcare systems requires intentional inquiry and strategic implementation. A successful Canadian quality improvement project, for example, recognized a gap in care, and increased MOUD access within a specific care delivery setting. The Best-Practice in Oral Opioid Agonist (BOOST) Collaborative in Vancouver, British Columbia, launched an 18-month quality improvement initiative in August 2017 to enhance access to MOUD in interprofessional community-based health centers. ${ }^{16}$ The initiative, grounded in the Institute for Healthcare Improvement's Breakthrough Series Collaborative methodology, ${ }^{17}$ had five predefined project quality indicators: (1) engagement in care (i.e., a documented encounter with a primary care provider within the prior 18 months), (2) OAT access (i.e., a documented OAT prescription), (3) active OAT (i.e., a non-expired OAT prescription), (4) retention in care (i.e., 3 months or longer), and (5) a quality of life score. ${ }^{16}$ The BOOST Collaborative's online and in-person training programs translated OAT best practices into clinical practice through teaching and implementing plan, do, study, act cycles (PDSAs), an improvement method for testing small changes in the clinical setting that facilitates teaching and learning opportunities. ${ }^{16}$ Preliminary results from quarter 4 reporting in December 2019 are promising. 
By this time period the program enrolled 29 teams, reached 4601 clients, $79 \%$ of clients had an active OAT prescription, $88 \%$ of clients were retained on OAT for greater than 3 months, and $84 \%$ participating teams engaged clients and family voice in their work. $^{18}$

Similarly, the VHA is implementing and evaluating a program intended to enhance MOUD access in non-substance use disorder (SUD) specialty treatment settings through the Stepped Care for Opioid Use Disorder Train-the-Trainer (SCOUTT) Program. ${ }^{19}$ The SCOUTT Program trains providers to increase MOUD access in non-SUD VHA care delivery settings (e.g., primary care) and facilitates implementation through a learning collaborative across $18 \mathrm{VHA}$ regional networks. ${ }^{20}$

The focus on MOUD access gaps in the outpatient setting, as seen in the BOOST Collaborative and SCOUTT Programs, is important. However, health system leaders should consider MOUD access through other entry points within the conventional healthcare delivery system. Specifically, access to MOUD in acute care settings (e.g., hospitalization or emergency department visits). We recently observed that OAT delivery during acute hospitalization for patients with OUD within the VHA system was rare. ${ }^{21}$ Only $15 \%$ of eligible VHA patients received OAT during admission, and hospitals varied in OAT delivery- 0 to $43 \%$ of qualified admissions. ${ }^{21}$

Outside the VHA, there is a growing focus on MOUD delivery in the hospital with the introduction of addiction consult services (ACSs) composed of transdisciplinary teams (e.g., physicians, nurse practitioners, social workers, SUD counselors, peers, physician assistants, and trainees). ${ }^{22}$ The ACSs manage addiction care for people hospitalized with SUDs most often for health reasons secondary to their SUD. Teams provide medically specific care to people with SUD including medication, pain management, conflict resolution, harm reduction, and warm-hand offs to outpatient care. ${ }^{22}$ Additionally, to address care continuity issues at hospital discharge, low-barrier bridge clinics are an emergent clinical delivery intervention to facilitate the transition of patients from the inpatient setting to outpatient programming. ${ }^{23}$ Bridge clinics may include MOUD, harm reduction programming, and peer support services. Another potential clinical touchpoint for MOUD within the acute care system is the emergency department (ED). Some EDs provide buprenorphine, ${ }^{24}$ in addition to other services like peer support. ${ }^{25}$ Interestingly, Massachusetts passed a law in 2018 requiring all of its EDs to offer MOUD to all patients presenting with an OUD. ${ }^{26}$

\section{ELEMENT 3. BROADEN THE DEFINITION OF THE MOUD DELIVERY SYSTEM}

The definition of the twenty-first century MOUD delivery system is evolving. A National Academies of Sciences, Engineering, and Medicine report described MOUD delivery in a variety of clinical and non-clinical settings: office-based opioid treatment; opioid treatment programs (OTPs); acute care settings (e.g., hospital); other care settings (e.g., homeless shelters); correctional facilities (e.g., jail); innovative settings (e.g., mobile medication units); and low-barrier medication-based treatment. ${ }^{27}$ Looking outside conventional clinical settings is important to meet the needs of people who feel unsafe in medicalized environments due to stigma and discrimination related to drug use, ${ }^{28,}{ }^{29}$ interpersonal, generational, and structural trauma; and/or complicating psychosocial factors (e.g., housing insecurity, serious mental illness). Clinical leaders should consider adopting an expanded view of the MOUD delivery system with potential treatment access points that include non-healthcare delivery settings, such as places of worship, ${ }^{30}$ street medicine programming, ${ }^{31}$ and colocating MOUD in harm reduction spaces (e.g., syringe exchange programs). ${ }^{32}$ Moreover, in the time of COVID-19, federal regulation changes allow buprenorphine initiation through telemedicine or audio-only communication. ${ }^{33}$ These changes rapidly expand potential delivery settings. A Rhode Island program, for example, launched a 24-hour telephone hotline to initiate patients on buprenorphine ${ }^{34}$ and another group, in Boston, MA, describes partnering with community-based outreach harm reduction specialists who facilitate telemedicine visits in the community with addiction physicians who subsequently prescribe buprenorphine. ${ }^{35}$ These types of programs are possible in the VHA as illustrated through a VHA pilot program, which used mobile technology and FaceTime, paired with an ongoing communitybased case management program to provide buprenorphine treatment for Veterans experiencing homelesssnes. ${ }^{36}$

\section{ELEMENT 4: EXPAND MOUD OPTIONS}

Finally, although legally and likely politically challenging, clinical leaders and researchers could consider other MOUD options, with the potential for research, and eventual expansion in the USA. Current US federal policy limits OAT choices to buprenorphine (oral, implant, and long-acting injectables) and OTP-dispensed methadone. Certain Canadian jurisdictions, in contrast, offer additional OAT options beyond methadone and buprenorphine including slowrelease oral morphine and injectable OAT (i.e., diacetylmorphine and hydromorphone). ${ }^{37}$ Note, injectable OAT in Canada is only recommended for patients with "severe, treatment-refractory opioid use disorder and ongoing illicit injection opioid use" who do not benefit from oral OAT. ${ }^{38}$ Furthermore, in Canada, methadone is dispensed through the pharmacy system. ${ }^{37}$ In addition to expanded MOUD options, British Columbia public health authorities launched a safe supply prescribing program in response to COVID-19. ${ }^{39}$ This program prescribes medicalgrade pharmaceutical products as an alternative substance supply for people with SUDs during COVID-19. ${ }^{39}$ For those with OUD, prescribing options include 12-hour oral morphine and/or oral hydromorphone. ${ }^{39}$ This is a novel 
public health intervention and the effectiveness and safety of this program is unknown and unstudied to date.

\section{CONCLUSION}

No singular solution addresses the drug-related overdose crisis. Increased access to MOUD, however, can mitigate harm. The four elements, supported by real-life exemplars, provide institutional and policy decisionmakers with potential pathways for extending and strengthening the MOUD care continuum. Policies, systems, and programs should be designed and co-created in partnership with patients and communities with lived experience. Patient and community preferences should not only be considered but prioritized in policy and service delivery design, including culturally specific needs for marginalized populations. Decisionmakers should identify local system gaps to develop a high-touch service delivery system in which patients can rapidly access MOUD in all conventional clinical settings. Clinical and public health leaders, moreover, should view the MOUD delivery system more broadly to include MOUD access points in non-clinical environments. Finally, although not legally possible in the USA, leaders can learn about a broader spectrum of OAT options provided in Canada, and elsewhere. MOUD is by no means a panacea to this crisis but is an important medical and public health intervention.

Corresponding Author: Kelsey C. Priest, PhD, MPH; School of Medicine, $M D / P h D$ Program, Oregon Health \& Science University, Portland, OR, USA (e-mail: priest@ohsu.edu).

Funding National Institute on Drug Abuse (F3O DAO4470O) and United States Department of Veterans Affairs Health Services Research \& Development (IK2HXO01516). Funding organizations were not involved in the design of the study, data collection, data analysis, the interpretation of data, or in the writing of the manuscript. The contents of the manuscript are those of the authors and do not represent the views of the U.S. Department of Veterans Affairs or the U.S. Government.

\section{Compliance with Ethical Standards:}

Conflict of Interest: Dr. McCarty presented at the Boost Collaborative launch on September 15, 2017, on quality improvement methodology. The Boost Collaborative reimbursed him for his travel expenses. He had no formal involvement with the program after his presentation.

\section{REFERENCES}

1. Finlay AK, Binswanger IA, Timko C, et al. Facility-level changes in receipt of pharmacotherapy for opioid use disorder: implications for implementation science. J Subst Abus Treat 2018;95:43-47.

2. Madras BK, Ahmad NJ, Wen J, Sharfstein J. Improving access to evidence-based medical treatment for opioid use disorder: strategies to address key barriers within the treatment system. NAM Perspectives 2020

3. Wyse JJ, Gordon AJ, Dobscha SK, et al. Medications for opioid use disorder in the Department of Veterans Affairs (VA) health care system: Historical perspective, lessons learned, and next steps. Subst Abus 2018;39(2):139-144.
4. James $\mathbf{K}$, Jordan A. The opioid crisis in black communities. The Journal of Law, Medicine \& Ethics 2018;46(2):404-421.

5. Lagisetty PA, Ross R, Bohnert A, Clay M, Maust DT. Buprenorphine treatment divide by race/ethnicity and payment. JAMA psychiatry 2019;76(9):979-981.

6. Tsai AC, Kiang MV, Barnett ML, et al. Stigma as a fundamental hindrance to the United States opioid overdose crisis response. PLoS Med 2019;16(11).

7. Manhapra A, Stefanovics E, Rosenheck R. Initiating opioid agonist treatment for opioid use disorder nationally in the Veterans Health Administration: who gets what? Subst Abus 2019;41(1):110-120.

8. Santana MJ, Manalili K, Jolley RJ, Zelinsky S, Quan H, Lu M. How to practice person-centred care: a conceptual framework. Health Expect 2018;21(2):429-440.

9. Marchand $\mathbf{K}$, Beaumont $\mathbf{S}$, Westfall $\mathbf{J}$, et al. Conceptualizing patientcentered care for substance use disorder treatment: findings from a systematic scoping review. Substance Abuse Treatment, Prevention, and Policy 2019;14(1):37.

10. Winograd RP, Presnall N, Stringfellow E, et al. The case for a medication first approach to the treatment of opioid use disorder. Am J Drug Alcohol Abuse 2019;45(4):333-340.

11. Substance Abuse and Mental Health Services Administration. The Opioid Crisis and the Black/African American Population: An Urgent Issue. 2020.

12. Tsemberis $\mathbf{S}$. Housing first: The pathways model to end homelessness for people with mental illness and addiction manual. European Journal of Homelessness. 2011;5(2).

13. Winograd RP, Wood CA, Stringfellow EJ, et al. Implementation and evaluation of Missouri's Medication First treatment approach for opioid use disorder in publicly-funded substance use treatment programs. J Subst Abus Treat 2020; 108:55-64.

14. Hansen H, Roberts SK. Two tiers of biomedicalization: Methadone, buprenorphine, and the racial politics of addiction treatment. Adv Med Sociol 2012;14(12):79-102.

15. U.S. Department of Veterans Affairs. Evaluating VA patient-centered care: patient, provider, and organizational views. https://www.queri.research. va.gov/national_partnered_evaluations/patient_care.cfm. Published 2018.

16. Beamish L, Sagorin Z, Stanley C, et al. Implementation of a regional quality improvement collaborative to improve care of people living with opioid use disorder in a Canadian setting. BMC Health Serv Res. 2019;19(1): 1-8.

17. The Institute for Healthcare Improvement. The breakthrough series: IHI's collaborative model for achieving breakthrough improvement. 2003.

18. British Columbia Centre for Excellence in HIV/AIDS and BOOST. Project Update: Q4 Oct-Dec 2019. Stop HIV/AIDs. http://stophivaids.ca/boostproject-updates/. Accessed 9/29/2020.

19. U.S. Department of Veteran Affairs. Evaluating the Implementation of the VA Stepped Care for Opioid Use Disorder Train-the-Trainer (SCOUTT) Program. https://www.queri.research.va.gov/national_partnered_evaluations/stepped-care-oud.cfm. Published 2019.

20. Gordon AJ, Drexler K, Hawkins EJ, et al. Stepped Care for Opioid Use Disorder Train the Trainer (SCOUTT) initiative: expanding access to medication treatment for opioid use disorder within Veterans Health Administration facilities. Subst Abus 2020;41(3):275-282.

21. Priest KC, Lovejoy TI, Englander H, Shull S, McCarty D. Opioid agonist therapy during hospitalization within the Veterans Health Administration: a pragmatic retrospective cohort analysis. J Gen Intern Med. 2020.

22. Priest KC, McCarty D. Role of the hospital in the 21st Century opioid overdose epidemic: the addiction medicine consult service. J Addict Med. 2019;13(2):104-112.

23. Snow RL, Simon RE, Jack HE, Oller D, Kehoe L, Wakeman SE. Patient experiences with a transitional, low-threshold clinic for the treatment of substance use disorder: a qualitative study of a bridge clinic. J Subst Abus Treat 2019.

24. D'Onofrio G, O'Connor PG, Pantalon MV, et al. Emergency departmentinitiated buprenorphine/naloxone treatment for opioid dependence: a randomized clinical trial. JAMA. 2015;313(16):1636-1644.

25. McGuire AB, Powell KG, Treitler PC, et al. Emergency departmentbased peer support for opioid use disorder: emergent functions and forms. J Subst Abus Treat 2020;108:82-87.

26. WBUR News \& Wire Services. Lawmakers send opioid bill to Baker's desk. https://www.wbur.org/commonhealth/2018/08/01/opioid-legislationto-governor. Published 2018. Accessed 9/29/2020.

27. National Academies of Sciences, Engineering, and Medicine. Medications for Opioid Use Disorder Save Lives. Washington, DC: The National Academies Press; 2019. 
28. McNeil R, Small W, Wood E, Kerr T. Hospitals as a 'risk environment': an ethno-epidemiological study of voluntary and involuntary discharge from hospital against medical advice among people who inject drugs. Social science \& medicine (1982) 2014;105:59-66.

29. Biancarelli DL, Biello KB, Childs E, et al. Strategies used by people who inject drugs to avoid stigma in healthcare settings. Drug Alcohol Depend 2019; 198:80-86.

30. Woodard S. Drug and alcohol treatment in Hartford supported by new program. Addiction Now. 2018. https://www.drugaddictionnow.com/ 2018/05/22/drug-and-alcohol-treatment-hartford-supported-new-program/. Published 5/22/2018.

31. Carter J, Zevin B, Lum PJ. Low barrier buprenorphine treatment for persons experiencing homelessness and injecting heroin in San Francisco. Addiction science \& clinical practice 2019;14(1):20.

32. Hood JE, Banta-Green CJ, Duchin JS, et al. Engaging an unstably housed population with low-barrier buprenorphine treatment at a syringe services program: Lessons learned from Seattle, Washington. Subst Abus 2019:1-9.

33. Priest KC. The COVID-19 pandemic: practice and policy considerations for patients with opioid use disorder. Health Affairs Blog. 2020.
34. Samuels EA, Clark SA, Wunsch C, et al. Innovation during COVID-19: improving addiction treatment access. J Addict Med. 2020.

35. Harris M, Johnson S, Mackin S, Saitz R, Walley AY, Taylor JL. Low barrier tele-buprenorphine in the time of COVID-19: a case report. $J$ Addict Med. 2020.

36. Iheanacho $\mathbf{T}$, Payne $\mathbf{K}$, Tsai J. Mobile, Community-based buprenorphine treatment for veterans experiencing homelessness with opioid use disorder: a pilot, feasibility study. The American Journal on Addictions. 2020.

37. Priest KC, Gorfinkel L, Klimas J, Jones AA, Fairbairn N, McCarty D. Comparing Canadian and United States opioid agonist therapy policies. Int J Drug Policy 2019;74:257-265.

38. Fairbairn N, Ross J, Trew M, et al. Injectable opioid agonist treatment for opioid use disorder: a national clinical guideline. CMAJ : Canadian Medical Association journal=journal de l'Association medicale canadienne. 2019;191(38):E1049-E1056

39. British Columbia Centre on Substance Use. Risk mitigation: in the context of dual public health emergencies. 2020.

Publisher's Note: Springer Nature remains neutral with regard to jurisdictional claims in published maps and institutional affiliations. 